\title{
Generalidades, manejos, cuidados y manifestaciones clínicas del SARS-CoV-2.
}

\section{Overview, management, care and clinical manifestations of SARS-CoV-2.}

\author{
José Eduardo Orellana-Centeno,* Verónica Morales-Castillo, ${ }^{\ddagger}$ Roxana Nayeli Guerrero Sotelo ${ }^{\S}$
}

RESUMEN

Se mantienen los estudios para describir los síntomas clínicos del COVID 19, ya se encuentran documentados a nivel general o sistémico la fiebre, dificultad para respirar, tos seca, fatiga, diarrea y algunos otros menos comunes que se llegan a presentar. La sintomatología se presenta en diferentes etapas que van desde asintomáticas hasta severa y crítica. Con un periodo de incubación de hasta 14 días y con un promedio de seis días que es el momento más común de presencia de los signos y síntomas. Algunas de las manifestaciones orales presentes son: pérdida de la sensación del sabor (ageusia), ausencia del olfato, resequedad de la boca.

Palabras clave: COVID 19, salud pública, manifestaciones clínicas orales.

\section{INTRODUCCIÓN}

S e mantienen los estudios para describir los síntomas clínicos del COVID-19, ya se encuentran documentados a nivel general o sistémico la fiebre, dificultad para respirar, tos seca, fatiga, diarrea y alguno otros menos comunes que se llegan a presentar. La sintomatología se presenta en diferentes etapas que van desde asintomáticas hasta severa y crítica. Con un periodo de incubación de hasta 14 días y con un promedio de seis días que es el momento más común de presencia de los signos y síntomas. El modo de transmisión es por medio de contacto directo e indirecto por medio de gotas de Flügge por vías
ABSTRACT

Studies are ongoing to describe the clinical symptoms of COVID-19, and fever, shortness of breath, dry cough, fatigue, diarrea, and some other less common ones that have been reported are already documented at a general or systemic level. Symptomatology occurs at different stages ranging from asymptomatic to severe and critical. With an incubation period of up to fourteen days and with an average of six days, which is the most common moment of presence of signs and symptoms. Some of the oral manifestations present are: Loss of taste sensation (ageusia), absence of olfaction, dry mouth.

Keywords: COVID 19, public health, oral clinical manifestations.

\footnotetext{
* Maestro en Salud Pública. Profesor Investigador de tiempo completo. Universidad de la Sierra Sur, Instituto de Investigación Sobre la Salud Pública, Licenciatura de Odontología. Oaxaca, México.

‡ Médico Especialista en Medicina Familiar. Unidad Académica Multidisciplinaria Zona Media de la Universidad Autónoma de San Luis Potosí, Maestría en Administración. San Luis Potosí, México.

$\S$ Doctora en Estudios Sociales. Profesor Investigador tiempo completo. Universidad de la Sierra Sur, Instituto de Investigación Sobre la Salud Pública, Licenciatura de Enfermería. Oaxaca, México.
}

respiratorias superiores. Este tipo de contactos y vías son formas comunes de contaminación e infección en profesiones de la salud como es los odontólogos que están en alto riesgo potencial debido a que los procedimientos utilizados generan aerosoles que se asocian con la transmisión de infecciones respiratorias agudas. ${ }^{1,2}$

El SARS-CoV-2 no solamente se transmite por los aerosoles, también por el contacto con equipo, instrumental y mobiliario dental en donde el virus puede permanecer hasta 72 horas dependiendo del material que se trate. Los procedimientos de limpieza y desinfección que se hacen de manera rutinaria deben seguirse y aumentar las preocupaciones para evitar infecciones con las sustancias 
y químicos recomendados por la Center Disease Control (CDC), Asociación Dental Americana (ADA) y otras organizaciones de salud. ${ }^{3,4}$

\section{MANEJO ODONTOLÓGICO}

El personal de salud debe tomar decisiones para proporcionar un tratamiento, no basta el juicio profesional, sino que se alinea al cumplimiento de requerimientos y recomendaciones emitidas por autoridades a nivel local y mundial. Al encontrarnos en medio de una pandemia como es el COVID-19, en el ámbito mundial se considera que los tratamientos odontológicos hayan sido suspendidos exceptuando las urgencias. En el caso de tratamientos de emergencia, el odontólogo deberá dar tratamiento en su consultorio tomando todas las precauciones necesarias para evitar el riesgo de una infección. Los odontólogos debemos estar familiarizados con aquellos tratamientos en los que se requiera el uso de la pieza dental (alta y baja) que es un importante generador de aerosoles. ${ }^{5}$

El odontólogo debe empezar a utilizar cubrebocas N95 para su protección, así como también la correcta colocación y retiro de los mismos. Adicionalmente, el uso de guantes de látex o de nitrilo, gorro desechable, batas desechables con repelente preferentemente, gafas protectoras y careta. ${ }^{6}$

La ADA proporcionó a los odontólogos tres diferentes algoritmos para tomar decisiones del triaje de los posibles pacientes con COVID y con ello minimizar los riesgos de transmisión para el personal odontológico. Debemos considerar que el paciente infectado puede ser asintomático y por tanto el manejo que realicemos deberá ser preventivo. El uso de dique de hule es una importante herramienta para evitar la contaminación del campo de trabajo y también para la protección del odontólogo ante el posible contacto de sangre y saliva. Está comprobado que el uso del dique de hule disminuye el paso de partículas de aerosol emitidos por instrumentos como piezas dentales de alta y de baja. Otra de las recomendaciones emitidas es el manejo de consultorios que utilizan aislamiento de presión negativa con filtración de aire particulado de alta eficiencia, sobre todo en estos momentos de desconocimiento de la magnitud del SARS-CoV-2 y tomando en cuenta la necesidad de atención dental. ${ }^{4-7}$

Uno de los principales motivos de asistencia en adultos es el dolor causado por una infección dental provocado principalmente por caries, por tal motivo se le brinda atención con antibioticoterapia y en el caso de los pacientes infantiles no se presentan solamente por infección sino por caries, que aunque no se considera una causal de urgencia, los pacientes infantiles llegan a presentar molestias que pudieran confundir al padre. Los tratamientos que se pudieran realizar para atender al paciente pediátrico sin riesgo ante la presencia de caries son: técnica de restauración atraumática (TRA), aplicación de fluoruro diamino de plata, selladores de fosetas y fisuras y técnica de Hall. ${ }^{8}$

\section{MEDICAMENTOS}

Algunos de los antivirales que se utilizan para el tratamiento del coronavirus, como lopinavir y ritonavir, que son empleados en pacientes inmunocomprometidos con $\mathrm{VIH}$, pueden afectar la cavidad oral, tracto gastrointestinal, en boca se presentan estomatitis, úlceras bucales y sequedad en la cavidad bucal. ${ }^{9}$

Los esteroides sistémicos y tópicos son utilizados en el tratamiento de enfermedades con afectación bucal como son: pénfigo, liquen plano, penfigoide. Estos medicamentos pueden provocar una exacerbación potencial en pacientes afectados por SARS-CoV-2, por lo cual se aconseja suspendan dicha terapia. ${ }^{10}$

La cloroquina es un medicamento que también se utiliza contra el SARS-CoV-2 y para el tratamiento de enfermedades como el lupus eritematoso, dicha afección causa algunas manifestaciones en la cavidad bucal. ${ }^{11}$

Pacientes que están tomando medicamentos antiinflamatorios debido a la artritis reumatoide ocasionalmente presentan síndrome de Sjögren y son considerados medicamentos para aumentar el riesgo de complicaciones de COVID-19. La expresión de ACE2 proporciona rutas ventajosas para la entrada del virus, pacientes con diabetes, hipertensión y enfermedades cardiovasculares con prescripción médica deben inhibir ECA y reducir los bloqueadores Ang II-AT1, ya que estos medicamentos aumentan ECA2. ${ }^{12}$

$\mathrm{El}$ ingreso del SARS-CoV-2 ingresa por el receptor ACE2 que se encuentra en tejidos de la mucosa oral, especialmente en la lengua, piso de la boca y en el epitelio gingival, por lo cual es posible pensar que la cavidad bucal es un sitio de ingreso del COVID-19. La entrada del virus por la boca puede llevarse a cabo por las manos sucias ingresando a la boca, comida contaminada o el compartir vasos y platos de personas infectadas. ${ }^{13,14}$

Se reporta en literatura que la saliva es un importante elemento diagnóstico para el SARS-CoV-2, siendo mayor su detección por esta muestra biológica que la sanguínea. ${ }^{15}$ 


\section{MANIFESTACIONES CLÍNICAS}

\section{Generales: $^{15}$}
a) Fiebre
b) Tos seca
c) Sensación de falta de aire y dificultad para respirar
d) Fatiga
e) Dolor muscular
f) Dolor de cabeza
g) Dolor de garganta
h) Confusión
i) Diarrea
j) Vómito

\section{Orales: ${ }^{6,16}$}

a) Pérdida de la sensación del sabor (ageusia)

b) Ausencia del olfato

c) Resequedad de la boca

\section{BIBLIOGRAFÍA}

1. Carlos WG, Dela Cruz CS, Cao B, Pasnick S, Jamil S. Novel Wuhan (2019-nCoV) coronavirus. Am J Respir Crit Care Med. 2020; 201 (4): P7-P8. doi: 10.1164/rccm.2014P7.

2. Huang C, Wang Y, Li X, Ren L, Zhao J, Hu Y et al. Clinical features of patients infected with 2019 novel coronavirus in Wuhan, China. Lancet. 2020; 395: 497-506. doi: 10.1016/S01406736(20)30183-5.

3. Kohn WG, Collins AS, Cleveland JL, Harte JA, Eklund KJ, Malvitz DM; Centers for Disease Control and Prevention. 2003. Guidelines for infection control in dental health-care settings-2003. Available in: https://www.cdc.gov/mmwr/preview/mmwrhtml/rr5217a1.htm.

4. American Dental Association 2020b. ADA interim guidance for minimizing risk of COVID-19. Available in: transmission. https://www.ada.org/ /media/CPS/Files/COVID/ADA_COVID_ Int_Guidance Treat_Pts.pdf?utm_source $=$ cpsorg\&utm medium $=$ covid- - $p$ s $=$ virus-Ip\&utm_content $=$ cv-pm-ebd-interimresponse\&utm_campaign $=$ Covid $-1 \overline{9}$

5. Sabino-Silva R, Jardim ACG, Siqueira WL. Coronavirus COVID-19 impacts to dentistry and potential salivary diagnosis. Clin Oral Investig. 2020; 24 (4): 1619-1621. doi: 10.1007/s00784-02003248-x.

6. Dziedzic A, Wojtyczka R. The impact of coronavirus infectious disease 19 (COVID-19) on oral health [published online ahead of print, 2020 Apr 18]. Oral Dis. 2020; 10.1111/odi.13359. doi: 10.1111/odi.13359.

7. Samaranayake LP, Reid J, Evans D. The efficacy of rubber dam isolation in reducing atmospheric bacterial contamination. ASDC J Dent Child. 1989; 56 (6): 442-444.

8. Mallineni SK, Innes NP, Raggio DP, Araujo MP, Robertson MD, Jayaraman J. Coronavirus disease (COVID-19): characteristics in children and considerations for dentists providing their care. Int J Paediatr Dent. 2020; 30: 245-250. doi: 10.1111/ipd.12653.

9. Chu CM, Cheng VC, Hung IF, Wong MM, Chan KH, Chan KS, HKU/ UCH SARS Study Group. Role of lopinavir/ritonavir in the treatment of SARS: Initial virological and clinical findings. Thorax. 2004; 59: 252-256. doi: 10.1136/thorax.2003.012658.

10. Dong L, Hu S, Gao J. Discovering drugs to treat coronavirus disease 2019 (COVID-19). Drug Discov Ther. 2020; 14 (1): 58-60. doi: 10.5582/ddt.2020.01012.

11. Gao J, Tian Z, Yang X. Breakthrough: Chloroquine phosphate has shown apparent efficacy in treatment of COVID-19 associated pneumonia in clinical studies. Biosci Trends. 2020; 14 (1): 72-73. doi: 10.5582/bst.2020.01047.

12. Monteil V, Kwon H, Prado P, Hagelkrüys A, Wimmer RA, Stahl M et al. Inhibition of SARS-CoV-2 infections in engineered human tissues using clinical-grade soluble human ACE2. Cell. 2020; 181 (4): 905-913.e7. doi: 10.1016/j.cell.2020.04.004.

13. Wu Z, McGoogan JM. Characteristics of and important lessons from the coronavirus disease 2019 (COVID-19) outbreak in china: summary of a report of 72314 cases from the chinese center for disease control and prevention [published online ahead of print, 2020 Feb 24]. JAMA. 2020; 10.1001/jama.2020.2648. doi: 10.1001/jama.2020.2648.

14. Zhang H, Zhou P, Wei Y, Yue H, Wang Y, Hu M et al. Histopathologic changes and SARS-CoV-2 immunostaining in the lung of a patient with COVID-19. Ann Intern Med. 2020; M20-0533. doi: 10.7326/ M20-0533.

15. Chan JFW, Yuan S, Kok KH, To KKW, Chu H, Yang J et al. A familial cluster of pneumonia associated with the 2019 novel coronavirus indicating person-to-person transmission: a study of a family cluster. Lancet. 2020; 395: 514-523. doi: 10.1016/S0140-6736(20)30154-9.

16. Bi Q, Wu Y, Mei S et al. Epidemiology and transmission of COVID-19 in Shenzhen China: analysis of 391 cases and 1,286 of their close contacts. Med Rxiv. 2020. Available in: https://doi. org/10.1101/2020.03.03.20028423

Correspondencia:

José Eduardo Orellana Centeno

Universidad de la Sierra Sur.

Guillermo Rojas Mijangos s/n, esq. Av. Universidad,

Col. Ciudad Universitaria, 70800,

Miahuatlán de Porfirio Díaz, Oaxaca, México.

E-mail: orellana17@msn.com 